\title{
Superconducting Magnetic Energy Storage (SMES) Stabilizer Based Model Predictive Control Technique
}

\author{
M. Roshdy ${ }^{1}$, Ashraf M. Hemeida ${ }^{2}$, Abd-Allah A. Ibrahim ${ }^{3}$, Gaber Elsaady ${ }^{4}$ \\ 1, 2 Energy Engineering Faculty, Aswan University, Egypt \\ 3 Engineering Faculty, Aswan University, Egypt \\ 4 Engineering Faculty, Assiut University, Egypt
}

\begin{abstract}
This paper presents a design of Model predictive control (MPC) based superconducting magnetic Energy Storage (SMES) unit. Model predictive control (MPC) technique has the ability to consider the overall system constraints. The MPC technique is applied successfully for improving the overall power systems performance. The paper presents the application of MPC technique based SMES device for enhancing power system stabilization. A single-machine infinite bus power system model is used to examine the feasibility of applying MPC technique based SMES device for improving the power systems performance and damping power systems oscillations. The obtained results show that the proposed controller maintain the robust performance, minimize the effect of disturbances and specified uncertainties, very effectively.

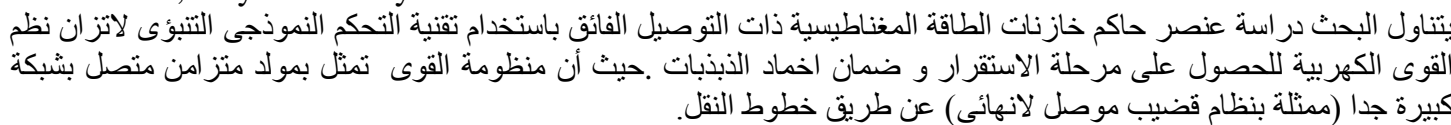

Keywords:

Single-machine infinite bus (SMIB), Superconducting Magnetic Energy Storage (SMES), Model predictive control (MPC), Proportional-Integral-Derivative Controller (PID).

\section{Nomenclature}

$T_{d 0}^{\prime}$ : d-axis transient open circuit time constant.

$\mathrm{H}$ : Inertia constant

$\mathrm{K}_{\mathrm{d}}$ : Damping coefficient

$\Delta \omega$ : Generator speed deviation

$\Delta \delta:$ Rotor angle deviation

$\Delta E_{q}^{\prime}$ : Transient internal voltage deviation

$\Delta \mathrm{E}_{\mathrm{fd}}$ : field voltage deviation

$\omega_{\mathrm{B}}$ : Rated speed in ele. Rad/sec

$\mathrm{V}_{\text {ref }}$ : Reference voltage

$\mathrm{V}_{\mathrm{t}}$ : Generator terminal voltage

$\mathrm{K}_{\mathrm{P}}$ : The proportional gain

$\mathrm{K}_{\mathrm{I}}$ : The integral gain

$\mathrm{K}_{\mathrm{D}}$ : the derivative gain

\section{Introduction.}

Model predictive control (MPC) is one of the most advanced technologies nowadays. The main advantage of MPC is its ability to consider system constraints implicitly. Due to this advantage, MPC is also widely used in power systems, such as voltage control $[1,2]$ and frequency regulation.

An integrated power management for a plug-in hybrid electric vehicle (PHEV) with multiple energy sources was presented including a semi-active hybrid energy storage system (HESS) and an assistance power unit (APU). In the integrated control strategy, the model predictive control strategy regulates the output power between the battery packs and ultra- capacitor packs, while the rule-based strategy allocates the output power between the APU and HESS. The fuel economy improvement under the MANHATTAN driving cycle is $21.88 \%$ [3]. The joining between fuzzy model and predictive control utilizes to beat the control problems of the super heater steam temperature (SST) of the power plant, likewise they have the feasibility and effectiveness [4]. MPC is classified to centralize, distribute and hierarchical which use as a viable solutions to damp wide-area electromechanical oscillations in largescale power systems. The hierarchical scheme gives the best performances and robustness [5]. The load frequency control (LFC) problem of multi-area 
interconnected power system is a disturbance rejection problem of large-scale system with state and input constraints, thus the distributed model predictive control (DMPC) technique is applied for this type of control problem. The overall system is resolved into several subsystems and each has its own local area MPC controller. The scheme awards the robust performance while respecting physical severe restrictions [6]. As well as, decentralized MPC is used to reduce the effect of the uncertainty due to governor and turbine parameters variation and load disturbance [7]. In Smart Grid, advanced plug-in electric vehicles (PEVs) can be connected with the distribution system, however the charging load can't be predicted. Then, it is complicated the operations of distribution systems. MPC based power dispatch approach is employed to minimize the operational cost while accommodating the PEV charging uncertainty [8]. The (D-MPC) of a wind farm equipped with fast and short-term energy storage system (ESS) for optimal active power control using the fast gradient method via dual decomposition. The wind farm size doesn't affect on this algorithm so, it is suitable for the modern wind farm application [9]. An MPC is designed for controlling the frequency of wind-penetrated power systems. MPC is robust which uses the knowledge of the estimated worst-case power imbalance [10]. The model predictive control approach is applied to a photovoltaic-diesel-battery hybrid system to optimally dispatch uses of its components. The performances of the closed-loop system are satisfactory [11]. Nonlinear MPC applied in a hybrid power system including a direct methanol fuel cell (DMFC) and a lithium-ion battery, then provides a good efficiency and robustness [12]. A distributed two-level cooperative model predictive control scheme has been presented for the power output regulation in a distributed system with a group of PV generators [13]. A railway traction power conditioner (RTPC) based on cascaded multilevel structure was presented for power quality compensation in high-speed railway system. An improved MPC strategy for RTPC was proposed to minimize current tracking errors and reduce the requirement of control frequency of MPC [14]. The explicit MPC was applied for frequency control in an actual isolated power system in Inner Mongolia. The system frequency can be restored by EMPC to its nominal value under large disturbance [15]. MPC was used for a grid-connected inverter in a photovoltaic system. The proposed strategy was effectiveness and validated [16].

This paper presents a design of Model predictive control (MPC) based superconducting magnetic Energy Storage (SMES) unit. Model predictive control (MPC) technique has the ability to consider the overall system constraints. The MPC technique is applied successfully for improving the overall power systems performance. The paper presents the application of MPC technique based SMES device for enhancing power system stabilization. A singlemachine infinite bus power system model is used to examine the feasibility of applying MPC technique based SMES device for improving the power systems performance and damping power systems oscillations. The obtained results show that the proposed controller maintain the robust performance, minimize the effect of disturbances and specified uncertainties, very effectively.

\section{SYSTEM STUDIED}

Fig. 1 shows schematic of the system studied, which a single machine infinite bus (SMIB) power system is considered [17]. The SMIB system is called the plant which consists of a synchronous generator connected through transmission line to a very large power network approximated by an infinite bus. The synchronous generator is driven by a turbine with a governor and excited by an external excitation system. The excitation system is controlled by an automatic voltage regulator (AVR) and a PID controller. The power system considered in this study is the fourth order linearized one-machine and infinite bus system [17].

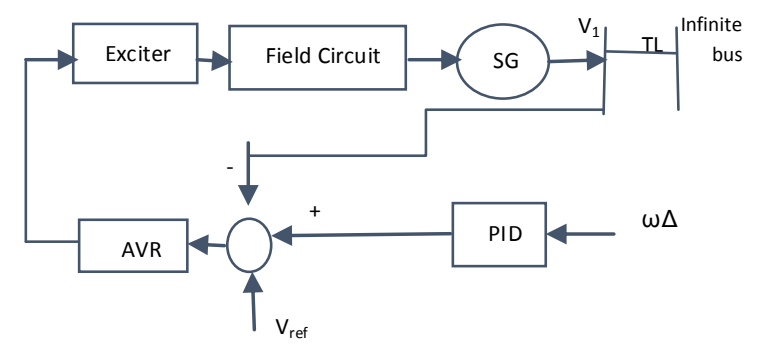

Fig 1. Schematic of a single-machine infinite-bus (SMIB) power system.

Fig. 2 shows a block diagram of transfer functions describing the different subsystems of the one machine infinite bus power system. The different subsystems blocks are given as [18];

A. Excitation system:

$$
\frac{K_{E}}{1+s T_{E}}
$$

Where, $\mathrm{K}_{\mathrm{E}}$ is the gain of exciter and $\mathrm{T}_{\mathrm{E}}$ is time constant of exciter.

B. Field flux decay:

$$
\frac{K_{3}}{1+s K_{3} T_{d 0}^{\prime}}
$$


Where, $T_{d 0}^{\prime}$ is the d-axis transient open circuit time constant.

C. Machine mechanical dynamics loop:

$$
\frac{1}{2 H s+K_{d}}
$$

Where, $\mathrm{H}$ is the inertia constant and $\mathrm{K}_{\mathrm{d}}$ is damping coefficient.

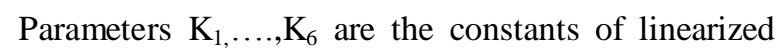
model of synchronous machine. From the block diagram, shown in Fig. 2, and using Eqs. (1, 2 and 3) the following fourth order linearized one machine infinite bus system can be derived as described in [17, $18]$.

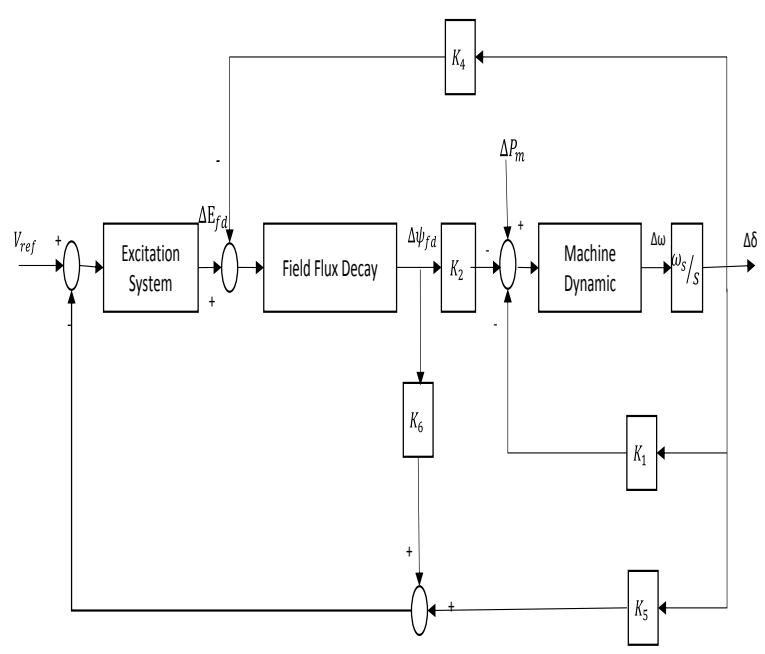

Fig 2. Block diagram of one machine infinite bus system

The values of $\mathrm{K}_{1}: \mathrm{K}_{6}$ are calculated according to the operating conditions of the generation system and connected power System [18]. Details of these constants are given in Appendix II.

\section{Superconducting Magnetic Energy Storage (SMES):}

A SMES system requires Power Conversion System (PCS) that can be either a voltage or current source inverter. Since the inverter utilized for a FACTS controller is voltage source type. The integration of SMES and FACTS devices can provide independent real and reactive power absorption or injection. If a transmission line experiences significant power transfer variations, SMES can be installed to relieve the loaded transmission line. A SMES system consists of a superconducting coil, cryogenic system and the power conversion or conditioning system (PCS) used for control and protection functions. IEEE defines SMES as - SMES device containing electronic converters that rapidly injects and/or absorbs real and/or reactive power or dynamically controls power flow in an ac system [19].

The SMES device is used to damp the oscillations due to load disturbances that must be controlled. The control signal of SMES device is the frequency deviation at bus which SMES is connected. However, the output power from SMES, absorbed or injected to the connected bus, is controlled. Fig. 3 shows the control system of SMES FACTS device.

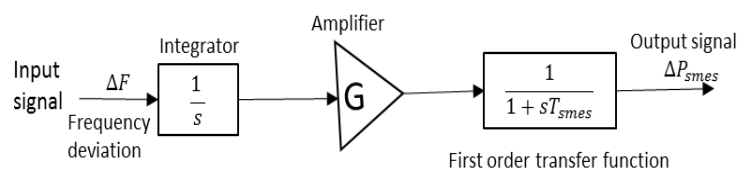

Fig 3. Control system of SMES and FACTS devices

The value of gain block $G$ of control system is selected to be 6 by trial and error, Also the SMES device is simulated as first order transfer function by time constant Tsmes of $0.03 \mathrm{sec}$.

\section{Continuous Time PID Controller Model :}

The PID controller is straightforward and simple to execute. It is broadly applied in industry to solve different control problems. PID controllers are utilized for decades. During this time, numerous adjustments have been presented in the literature [2021]. Then the transfer function of the modified continuous time PID controller is given by [22]:

$$
P I D(s)=K_{P}+K_{I} \frac{1}{s}+K_{D} \frac{s}{\tau s+1}
$$

Where, $K_{P}$ is the proportional gain, $K_{I}$ is the integral gain, $K_{D}$ is the derivative gain and the term $\frac{1}{\tau s+1}$ acts as an effective low-pass filter on the D regulator to attenuate noise in the derivative block. The individual effects of these three terms on the closed loop performance are summarized in [22].PID controller parameters are determined from the Matlab tuning given by [22]:

$K_{P}=15.5, K_{I}=5, K_{D}=0.0115, \tau=0.01$

Where, the speed deviation $\Delta \omega$ is the input to the PID controller, and the filter is used to remove the controller effect at steady state conditions.

\section{Model Predictive Control Technique :}

The MPC has proved to efficiently control an extensive variety of utilizations in industry, for example, chemical process, petrol industry, electromechanical systems and numerous different applications. The MPC scheme depends on an explicit use of a prediction model of the system response to obtain the control actions by minimizing an objective function. Optimization objectives include minimization of the difference between the 
predicted and reference response, and the control effort subjected to prescribed constraints. The effectiveness of the MPC is demonstrated to be proportionate to the optimal control. It shows its main strength in its computational expediency, realtime applications, intrinsic compensation for time delays, treatment of constraints, and potential for future extensions of the methodology. At each control interval, the first input in the optimal sequence is sent into the plant, and the entire calculation is repeated at subsequent control intervals. The purpose of taking new measurements at each time step is to compensate for unmeasured disturbances and model inaccuracy, both of which cause the system output to be different from the one predicted by the model $[23,24]$.

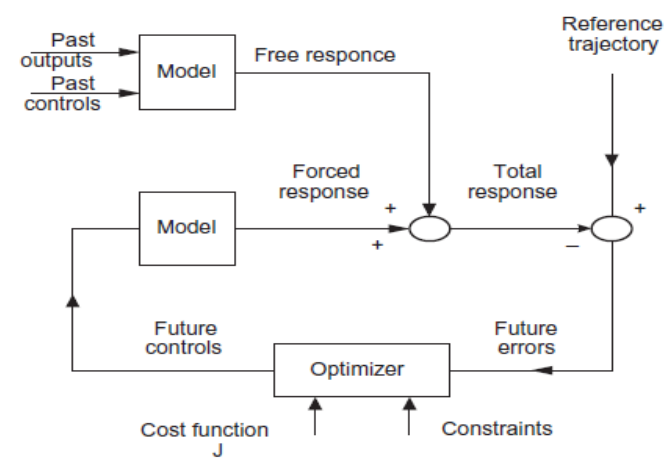

Fig 4. A simple structure of the MPC controller

Fig 4. shows a simple structure of the MPC controller. An internal model is used to predict the future plant outputs based on the past and current values of the inputs and outputs and on the proposed optimal future control actions. The prediction has two main components: the free response which being expected behavior of the output assuming zero future control actions, and the forced response which being the additional component of the output response due to the candidate set of future controls. For a linear system, the total prediction can be calculated by summing both of free and forced responses; reference trajectory signal is the target values the output should attain. The optimizer is used to calculate the best set of future control action by minimizing a cost function $(\mathrm{J})$, the optimization is subject to constraints on both manipulated and controlled variables [25]. The general objective is to tighten the future output error to zero, with minimum input effort. The cost function to be minimized is generally a weighted sum of square predicted errors and square future control values, e.g. in the Generalized Predictive Control (GPC):

$$
J\left(N_{1}, N_{2}, N_{u}\right)=\sum_{j=N_{1}}^{N_{2}} \beta(j)[\hat{y}(k+j / k)-w(k+j)]^{2}+\sum_{j=1}^{N_{u}} \lambda(j)[u(k+j-1)]^{2}
$$

Where, N1, N2 are the lower and upper prediction horizons over the output, $\mathrm{Nu}$ is the control horizon, $\beta(\mathrm{j}), \alpha(\mathrm{j})$ are weighting factors. The control horizon permits to decrease the number of calculated future control according to the relation: $\Delta \mathrm{u}(\mathrm{k}+\mathrm{j})=0$ for $\mathrm{j} \geq \mathrm{Nu}$.

The $w(k+j)$ represents the reference trajectory over the future horizon $\mathrm{N}$. Constraints over the control signal, the outputs and the control signal changing can be added to the cost function as follows:

$$
\begin{aligned}
& U_{\text {min }}=U(K) \leq U_{\text {max }} \\
& U_{\text {min }} \leq \Delta U(K) \leq \Delta U_{\text {max }} \\
& y_{\text {min }} \leq y(K) \leq y_{\text {max }}
\end{aligned}
$$

Solution of Eq. (5) gives the optimal sequence of control signal over the horizon $\mathrm{N}$ while respecting the given constraints of Eq. (6). The MPC technique has many advantages, in particularly it can pilot a big variety of process, being simple to apply in the case of multivariable system, can compensate the effect of pure delay by the prediction, inducing the anticipate effect in closed loop, being a simple technique of control to be applied and also offer optimal solution while respecting the given constraints. On the other hand, this type of restructure required the knowledge of model for the system, and in the present of constraints it becomes a relatively more complex regulator than a simple conventional controller such as a PID for example, and it takes more time for online calculations.

\section{SIMULATION RESULTS}

Computer simulation is carried out in order to validate the effectiveness of the proposed scheme. The Matlab/Simulink software package is used for this purpose.

Firstly, the proposed system is tested under the condition of step change in the mechanical torque by $10 \%$ as shown in Fig 5. 

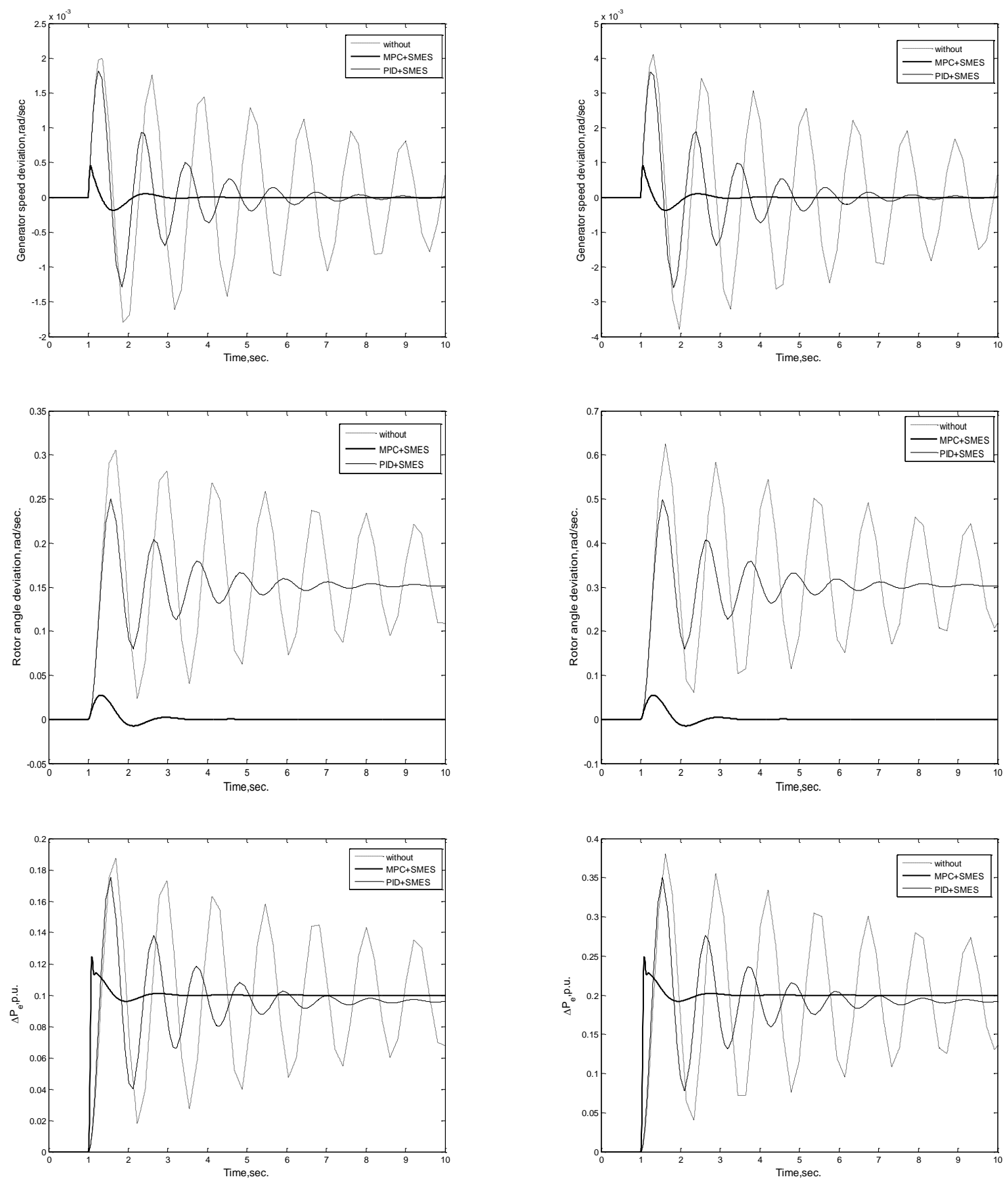

Fig 5. Dynamic responses to step change by $10 \%$ in the mechanical torque

Secondly, the proposed system is tested under the condition of step change in the mechanical torque by $20 \%$ as shown in Fig 6.

Fig 6. Dynamic responses to step change by $20 \%$ in the mechanical torque

Finally, the proposed system is tested under the condition of step change in the mechanical torque by 40\% as shown in Fig 7. 

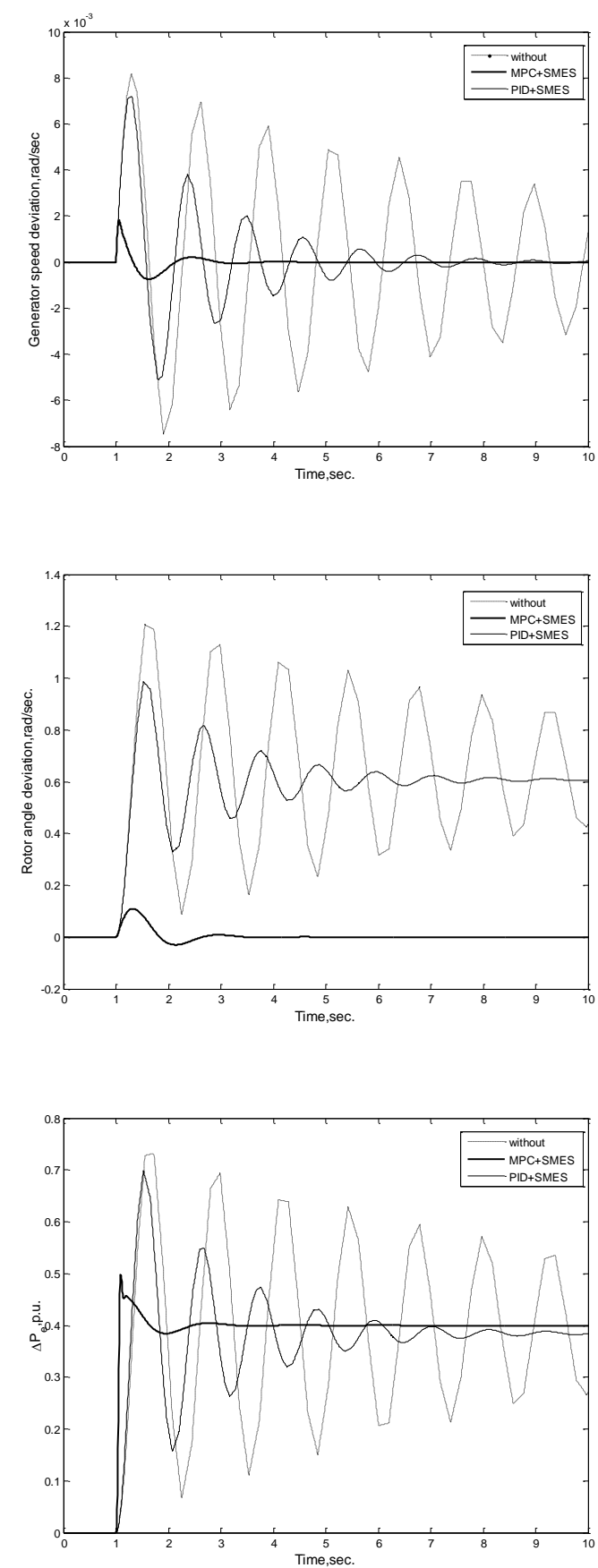

Fig 7. Dynamic responses to step change by $40 \%$ in the mechanical torque

From the figures, it is clear that the proposed controller (MPC) is very effective in damping the oscillation compared to other controllers.

\section{CONCLUSIONS}

This paper investigates Superconducting Magnetic Energy Storage (SMES) Stabilizer for single-machine infinite bus (SMIB) based on Model Predictive Control Technique. Digital simulations have been carried out in order to validate the effectiveness of the proposed scheme. A performance comparison between the proposed MPC and PID controllers has been carried out. The simulation results demonstrate that, the dynamic response of the system has been improved by using the MPC.

\section{REFERENCES}

1. Ma, H.M., Hill, D.J.: 'Adaptive coordinated voltage control - part I basic scheme', IEEE Trans. Power Syst., 2014, 29, (4), pp. 1546-1553

2. Ma, H.M., Hill, D.J.: 'Adaptive coordinated voltage control - part II use of learning for rapid response', IEEE Trans. Power Syst., 2014, 29, (4), pp. 1554-1561

3. Shuo Zhang "Model predictive control for power management in a plug-in hybrid electric vehicle with a hybrid energy storage system" Applied Energy 185 (2017) 1654-1662

4. Xiao Wu "Fuzzy Modeling and Predictive Control of Power plant Steam Temperature System “ IFAC-papers OnLine 48-30 (2015) 397-402

5. Da Wang" Comparison of centralized, distributed and hierarchical model predictive control schemes for electromechanical oscillations damping in large-scale power systems" Electrical Power and Energy Systems 58 (2014) 32-41

6. Hong Chen "Distributed model predictive load frequency control of multi-area interconnected power system" Electrical Power and Energy Systems 62 (2014) 289-298

7. T.H. Mohamed, H. evrani,"Decentralized model predictive based load frequency control in an interconnected power system" Energy Conversion and Management 52 (2011) 1208-1214

8. Jianhui Wang "Model predictive control-based power dispatch for distribution system considering plug-in electric vehicle uncertainty" Electric Power Systems Research 106 (2014) 29- 35

9. Haoran Zhao "Optimal active power control of a wind farm equipped with energy storage system based on distributed model predictive control" IET Gener. Transm. Distrib. 2016, Vol. 10, Iss. 3, pp. 669-677

10. Anne Mai Ersdal "Model Predictive Control for Power System Frequency Control Taking into Account Imbalance Uncertainty" Proceedings of the 19th World Congress the International Federation of Automatic Control Cape Town, South Africa. August 24-29, 2014 
11. Bing Zhu "Model Predictive Control for Energy Dispatch of a Photovoltaic-Diesel-Battery Hybrid Power System" Proceedings of the 19th World Congress the International Federation of Automatic Control Cape Town, South Africa. August 24-29, 2014

12. Martin Behrendt "Model predictive control of a hybrid fuel cell \& battery power system" 8th IFAC Symposium on Advanced Control of Chemical Processes The International Federation of Automatic Control Singapore, July 10-13, 2012

13. Fuwen Yang "Cooperative Model Predictive Control for Distributed Photovoltaic Power Generation Systems" IEEE Journal of Emerging and Selected Topics In Power Electronics, Vol. 04, No. 2, June 2016

14. Fujun Ma "Multilevel Power Conditioner and its Model Predictive Control for Railway Traction System" IEEE Transactions on Industrial Electronics, Vol. 63, No. 11, November 2016

15. Hao Jiang "Explicit model predictive control applications in power systems: an AGC study for an isolated industrial system "IET Generation, Transmission \& Distribution

16. Jiefeng $\mathrm{Hu}$ "Model Predictive Control of GridConnected Inverters for PV Systems With Flexible Power Regulation and Switching Frequency Reduction" IEEE Transactions on Industry Applications, Vol. 51, No. 1, January/February 2015

17. P. Kundur, "Power System Stability and Control”, New York: McGraw-Hill, 1994

18. G. Shabib, " Digital Design of a Power System Stabilizer for Power System Based on Plant-Input Mapping", International Journal of Electrical Power \& Energy Systems (IJEPES), Vol. 49, pp. 40-46, July 2013.

19. Sandeep Bhongade, H.O. Gupta, Barjeev Tyagi "Effect of SMES Unit in Multi-area Automatic generation control scheme suitable in restructured power system" International Journal of Distributed Energy Resources, vol. 7, no. 2, April-June 2011, pp. 149-165, Technology and Science Publishers, Germany.

20. H. Hjalmarsson, and T. Birkeland, "Iterative Feedback Tuning of Linear Time-Invariant Mimo Systems", In 37th IEEE Conference on Decision and Control, pp. 3893-3898, 1998.

21. G. Shabib, "Implementation of a Discrete Fuzzy PID Excitation Controller for Power System Damping", Ain Shams Engineering Journal (ASEJ), Vol. 3, Issue. 2, pp. 123-131,June 2012.

22. G. Shabib, Esam H. Abd-Elhameed, G. Magdy, "A New Approach to the Digital Implementation of Analog Controllers for a Power System Control", International Journal of Scientific \& Engineering Research (IJSER), Vol. 5, Issue 10, pp. 419-427, October-2014.

23. J. Thomas, D. Dumur, J. Buisson, H. Gueguen "Model predictive control for hybrid systems under a state partition based MLD approach (SPMLD)" International conference on informatics in control, automation and robotics ICINCO'04, vol.3, Setúbal; 2004. p. 78-85.

24. J. Richalet, A. Rault, J. L. Testud, J. Japon "Model predictive heuristic control, application to industrial processes" Automatic 1978;14(5):41328.

25. De Silva Clarence W. Mechatronic systems: devices, design, control, operation and monitoring, book, Taylor and Francis Group, LLC; 2008.

\section{APPENDIX I}

I.1 Generator parameters:

$\mathrm{H}=4.63, \mathrm{Kd}=4.4, \mathrm{~T}_{\mathrm{d} 0}^{\prime}=7.67, \omega_{\mathrm{B}}=377.0, \mathrm{X}_{\mathrm{d}}=0.973$

$\mathrm{pu}, \mathrm{x}_{\mathrm{d}}^{\prime}=0.19 \mathrm{pu}, \mathrm{X}_{\mathrm{q}}=0.55 \mathrm{pu}$

I. 2 Exciter parameters:

$\mathrm{K}_{\mathrm{e}}=50.0, \mathrm{~T}_{\mathrm{e}}=0.05$.

I.3 The K's:

$\mathrm{K}_{1}=0.5758, \mathrm{~K}_{2}=0.9738, \mathrm{~K}_{3}=0.6584, \mathrm{~K}_{4}=0.5266$,

$\mathrm{K}_{5}=-0.0494, \mathrm{~K}_{6}=0.8450$.

I.4 Transmission line:

$\mathrm{R}_{\mathrm{e}}=0.0, \mathrm{X}_{\mathrm{e}}=0.997 \mathrm{pu}$.

I.5 Operating point:

$\mathrm{Q}_{\mathrm{e} 0}=0.015 \mathrm{pu}, \mathrm{V}_{\mathrm{t} 0}=1.05 \mathrm{pu}, \mathrm{P}_{\mathrm{e} 0}=0.75 \mathrm{pu}$

\section{APPENDIX II}

The constants $\mathrm{K}_{1}: \mathrm{K}_{6}$ are evaluated with transmission line resistance $r_{e}=0$ given as follows [22]:

$$
\begin{aligned}
& \mathrm{K}_{1}=\frac{\mathrm{X}_{\mathrm{q}}-\mathrm{X}_{\mathrm{d}}}{\left(\mathrm{X}_{\mathrm{e}}+\mathrm{X}_{\mathrm{d}}^{\prime}\right)} \mathrm{I}_{\mathrm{q} 0} \mathrm{~V}_{0} \sin \delta_{0}+\frac{\mathrm{E}_{\mathrm{qo}} \mathrm{V}_{0} \cos \delta_{0}}{\left(\mathrm{X}_{\mathrm{e}}+\mathrm{X}_{\mathrm{q}}\right)} \\
& \mathrm{K}_{2}=\frac{\mathrm{V}_{0} \sin \delta_{0}}{\left(\mathrm{X}_{\mathrm{e}}+\mathrm{X}_{\mathrm{d}}^{\prime}\right)} \\
& \mathrm{K}_{3}=\frac{\mathrm{X}_{\mathrm{d}}^{\prime}+\mathrm{X}_{\mathrm{e}}}{\left(\mathrm{X}_{\mathrm{d}}+\mathrm{X}_{\mathrm{e}}\right)} \\
& \mathrm{K}_{4}=\frac{\mathrm{X}_{\mathrm{d}}-\mathrm{X}_{\mathrm{d}}^{\prime}}{\left(\mathrm{X}_{\mathrm{e}}+\mathrm{X}_{\mathrm{d}}^{\prime}\right)} \mathrm{V}_{0} \sin \delta_{0} \\
& \mathrm{k}_{5}=\frac{\mathrm{X}_{\mathrm{q}}}{\left(\mathrm{X}_{\mathrm{e}}+\mathrm{X}_{\mathrm{q}}\right)} \frac{\mathrm{V}_{\mathrm{d} 0}}{\mathrm{~V}_{\mathrm{t} 0}} \mathrm{~V}_{0} \cos \delta_{0}+\frac{\mathrm{X}_{\mathrm{d}}^{\prime}}{\left(\mathrm{X}_{\mathrm{e}}+\mathrm{X}_{\mathrm{d}}^{\prime}\right)} \frac{\mathrm{V}_{\mathrm{q} 0}}{\mathrm{~V}_{\mathrm{t} 0}} \mathrm{~V}_{0} \sin \delta_{0} \\
& \mathrm{~K}_{6}=\frac{\mathrm{X}_{\mathrm{e}}}{\left(\mathrm{X}_{\mathrm{e}}+\mathrm{X}_{\mathrm{d}}^{\prime}\right)} \frac{\mathrm{V}_{\mathrm{q} 0}}{\mathrm{~V}_{\mathrm{t} 0}}
\end{aligned}
$$

